OPEN ACCESS

Edited by:

Vincenzo Valentini,

Catholic University of the Sacred

Heart, Italy

Reviewed by:

Mariangela Massaccesi,

Fondazione Policlinico A. Gemelli

IRCCS, Italy

John Chetley Ford,

University of Miami, United States

*Correspondence:

Marcel Verheij

marcel.verheij@radboudumc.nl

Specialty section:

This article was submitted to

Radiation Oncology,

a section of the journal

Frontiers in Oncology

Received: 09 October 2020 Accepted: 16 December 2020 Published: 02 February 2021

Citation:

Hörner-Rieber J, Klüter S, Debus J, Adema G, Ansems M and Verheij M (2021) MR-Guided Radiotherapy: The

Perfect Partner for Immunotherapy?

Front. Oncol. 10:615697.

doi: 10.3389/fonc.2020.615697

\section{MR-Guided Radiotherapy: The Perfect Partner for Immunotherapy?}

\author{
Juliane Hörner-Rieber ${ }^{1,2,3,4}$, Sebastian Klüter ${ }^{1,2,3}$, Jürgen Debus ${ }^{1,2,3,4,5,6}$, Gosse Adema ${ }^{7}$, \\ Marleen Ansems ${ }^{7}$ and Marcel Verheij ${ }^{8 *}$ \\ 1 Department of Radiation Oncology, Heidelberg University Hospital, Heidelberg, Germany, ${ }^{2}$ Heidelberg Institute of Radiation \\ Oncology (HIRO), Heidelberg, Germany, ${ }^{3}$ National Center for Tumor Diseases (NCT), Heidelberg, Germany, ${ }^{4}$ Clinical \\ Cooperation Unit Radiation Oncology, German Cancer Research Center (DKFZ), Heidelberg, Germany, ${ }^{5}$ Heidelberg lon- \\ Beam Therapy Center (HIT), Department of Radiation Oncology, Heidelberg University Hospital, Heidelberg, Germany, \\ ${ }^{6}$ German Cancer Consortium (DKTK), Heidelberg, Germany, ${ }^{7}$ Radiotherapy \& Oncolmmunology Laboratory, Department of \\ Radiation Oncology, Radboud University Medical Center, Nijmegen, Netherlands, ${ }^{8}$ Department of Radiation Oncology, \\ Radboud University Medical Center, Nijmegen, Netherlands
}

During the last years, preclinical and clinical studies have emerged supporting the rationale to integrate radiotherapy and immunotherapy. Radiotherapy may enhance the effects of immunotherapy by improving tumor antigen release, antigen presentation, and T-cell infiltration. Recently, magnetic resonance guided radiotherapy (MRgRT) has become clinically available. Compared to conventional radiotherapy techniques, MRgRT firstly allows for daily on-table treatment adaptation, which enables both dose escalation for increasing tumor response and superior sparing of radiosensitive organs-at-risk for reducing toxicity. The current review focuses on the potential of combining MR-guided adaptive radiotherapy with immunotherapy by providing an overview on the current status of MRgRT, latest developments in preclinical and clinical radio-immunotherapy, and the unique opportunities and challenges for MR-guided radio-immunotherapy. MRgRT might especially assist in answering open questions in radio-immunotherapy regarding optimal radiation dose, fractionation, timing of immunotherapy, appropriate irradiation volumes, and response prediction.

Keywords: magnetic resonance-guided radiotherapy, adaptive treatment, immunotherapy, radioimmunotherapy, preclinical

\section{INTRODUCTION}

Over the last decades, substantial technical and methodological innovations in radiotherapy have enabled both more precise and focused delivery of higher doses of ionizing radiation combined with superior sparing of surrounding organs-at-risk (OARs). The latest development is magnetic resonance (MR)-guided radiotherapy (MRgRT), which bears the potential to revolutionize current standards and processes in radiotherapy. It not only offers superior soft-tissue contrast for precise detection of inter- and intrafractional changes in patient and tumor anatomy, but also allows for immediate reaction to these alterations by on-table plan adaptation (1-3). Thereby, safety margins can be reduced enabling dose escalation, while simultaneously limiting toxicity (4-7). Furthermore, some MR-linac devices offer gated dose delivery, which further facilitates irradiation of moving targets (8). Functional imaging, potentially integrated at the MR-linac, might allow for 
biologically guided radiotherapy to identify treatment responders, who could benefit from dose de-escalation, while additional (subvolume) boost dose might foster tumor control in non-responders $(9,10)$.

Despite tremendous advances in radiotherapy for improving local control and minimizing side-effects during the last decades, distant progression outside the irradiation field still remains a major challenge. Recently, immunotherapy has emerged as the fourth pillar in cancer treatment besides surgical resection, systemic therapy, and radiotherapy. Immunotherapy is increasingly regarded as a promising and attractive partner to radiotherapy, as ionizing radiation is known to inherit potent immunomodulatory effects by enhancing tumor immunogenicity and fostering immune-mediated tumor regression not only locally but also distant to the irradiation field $(11,12)$. However, for optimizing efficacy and reducing toxicity of anticancer radio-immunotherapy, redefinition of conventional radiotherapy volumes, doses, and fractionation schedules might be necessary $(13,14)$. Biologically individualized, MR-guided adaptive radio-immunotherapy might offer unique features to approach these challenges.

\section{CURRENT STATUS OF MR-GUIDED RADIOTHERAPY}

Hybrid systems for MRgRT, combining MR-scanners with radiotherapy devices, have first been proposed at the beginning of this century, and were introduced into clinical practice within the last years (15-17). Currently, two different systems are commercially available. Both make use of on-board magnetic resonance imaging (MRI) for patient positioning and enable treatment with step-and-shoot intensity modulated radiation therapy (IMRT) $(3,18,19)$. The systems also facilitate on-table treatment plan adaption based on the actual anatomic situation at the time of treatment. As the superior soft-tissue contrast of MRI allows for precise organ-at-risk delineation and therefore enables adaptive minimization of dose to normal tissue, it is expected that MRgRT will allow dose escalation (6).

With regard to targets susceptible to breathing motion, midposition based treatments using four-dimensional MRI acquired in treatment position directly at the MR-linac have been described (20), as well as real-time beam gating controlled by two-dimensional cine-MR $(8,21)$. Both strategies can contribute to a reduction of margins and thereby also potentially enable dose escalation.

First clinical data has been reported for various indications, and multiple clinical studies are ongoing that aim to show the benefits of this technology. Among others, the treated indications include liver $(22,23)$, pancreas $(22,24)$, lung $(5,25-28)$, prostate $(4,29-31)$, breast $(32,33)$, head and neck $(16,34)$, and oligometastatic disease $(7,35,36)$. Although MRguided treatments in principle can be performed in standard fractionation schemes, several authors report on the use of MRgRT for hypofractionated/stereotactic treatment schedules $(4,34,36-38)$ and even single fraction regimens $(39,40)$.
In addition, on-board MRI at MR-linacs can also be used for quantitative MRI, thereby potentially enabling treatment response monitoring as well as treatment plan adaption based on quantitative MRI information (9).

\section{CURRENT STATUS OF PRECLINICAL RADIO-IMMUNOTHERAPY}

Preclinical murine cancer models serve as an essential intermediate experimental model system to translate the findings from bench to bedside. In the radio-immunotherapy field, these models have extensively proven the high potential of combining radiotherapy with immunotherapy. Moreover, they have led to the identification of important underlying mechanisms. Preclinical evidence of synergy between radiotherapy and immune checkpoint blockade with anti-CTLA-4, anti-PD-1, or anti-PDL1 has been obtained in numerous murine models of cancer (41-46). Many of the challenges of combining radiation with immunotherapy (e.g. radiotherapy dose and fractionation schedule as well as sequence of therapy) have been investigated and show that both immunogenic and non-immunogenic radiation dose and schedules exist $(43,47)$. It is now wellestablished that immunogenicity is related to sensing of cytoplasmic DNA by the cGAS/STING (cyclic-GMP-AMP synthase/stimulator of interferon genes) pathway (44, 47-49). Although these preclinical studies have provided essential new insights into the potential of radio-immunotherapy, they also have limitations. Most studies combining radiotherapy and immunotherapy only use a single ablative dose or a hypofractionated radiotherapy schedule and as a consequence the optimal timing, dose, and treatment regimen vary between models and are difficult to compare. To investigate the abscopal effect of therapy, the majority of the preclinical studies use a transplantable cell line that is injected subcutaneously in two distant locations in the mouse. In these models one tumor is irradiated and the abscopal effects are monitored in the untreated secondary tumor. In contrast to human metastatic cancer lesions, the genetic and environmental factors in the primary and secondary tumor are almost identical. These models thus may not fully recapitulate human metastatic cancer. Moreover, many small animal studies still use large field, single-beam irradiation. In these platforms, radiation exposure has limited accuracy and precision. Moreover, in-depth investigation into the anti-tumor response may be hampered by high dose radiation to healthy tissue. Data from murine experiments are important but should be carefully interpreted and used in the translation to a clinical situation. The need for more precise radiation and a growing appreciation for the role of the tumor microenvironment in anti-tumor (immune) responses has led to major developments in small animal imaging technologies (including SPECT, CT, MRI). Combining these technologies with small animal radiation research platforms enables to better mimic modern radiotherapy practice (50). Several efforts have already led to the development of small animal image-guided radiation 
research platforms and showed their feasibility (51-56). Both for orthotopic (55) and genetically engineered mouse models of non-small lung cancer (51) preclinical image-guided radiotherapy platforms have been set up and demonstrated their feasibility to closely mimic clinical settings. Using a xenograft model of neuroblastoma, it was shown that small animal MRI-based radiotherapy planning not only allows for precision radiotherapy, but also for accurately measuring early tumor responses which are difficult to measure by calipers (54). Orthotopic mouse pancreatic tumors were treated with imageguided radiotherapy including treatment planning techniques comparable to patient treatment (52). Additionally, for spontaneous pancreatic tumors MRI guided radiotherapy platforms have been established (53).

To achieve the best predictive value of animal-based translational cancer research, models should provide biological mechanistic insights that can be tested in a clinical setting. This requires the availability of small animal image-guided radiotherapy platforms that evolve in line with advances in the clinic and suitable models in mice with a functional immune system that mimic human responses.

\section{CURRENT STATUS OF CLINICAL RADIO-IMMUNOTHERAPY}

In 1953, Mole et al. were the first to describe the so-called "abscopal effect" (from the Latin prefix $a b$ for "away from" and -scopus for "mark or target") for the immune-mediated regression of unirradiated tumor lesions at distance from the primary site of local radiotherapy (57). However, prospective evidence for the clinical efficacy of radio-immunotherapy is still limited today (58).

Initial data is especially found in the treatment of oligometastatic cancer patients. Four phase II trials have previously demonstrated that the addition of metastasisdirected ablative therapy for all tumor sites to standard of care treatment significantly improved at least progression-free survival (PFS) or even overall survival (OS) in several different tumor entities $(38,59-62)$. Two recently published phase II trials included metastatic non-small cell lung cancer (NSCLC) patients treated with the anti-PD-1 antibody pembrolizumab with or without locally ablative therapies including SBRT $(29,63)$. The study by Theelen et al. aimed to assess whether SBRT on a single tumor site preceding pembrolizumab could enhance tumor response to immunotherapy and reported a doubled overall response for the experimental arm as compared to immunotherapy only. Although PFS was more than three times and OS more than two times higher in the SBRT arm, no significance was reached. The observation that the largest effect occurred in the PD-L1-negative subgroup suggests that radiotherapy may increase the responsiveness of non-inflamed NSCLC tumors to immune checkpoint inhibition (63). This needs further clinical evaluation. The second trial by Bauml et al. included 51 oligometastatic NSCLC patients who had received locally ablative therapy to all known sites of disease and were additionally treated with pembrolizumab. Median PFS for the locally ablative therapy arm was significantly superior with 19.1 months compared to historical controls with only 6.6 months ( $\mathrm{p}=0.005)$ (29).

As most current studies on MR-guided adaptive radiotherapy focus on the treatment of oligometastases, the combination of immunotherapy with MRgRT of oligometastases appears especially attractive. Henke et al. recently published results of a phase I trial of MRgRT including oligometastatic tumor lesions of different origin, while others concentrated on MRgRT of adrenal, hepatic, lymph node, or bone metastases (7, 17, 23, 35, 64, 65). Radio-immunotherapy with daily MR-guided plan adaptation bears the potential to further reduce toxicity and improve local control, while simultaneous immunotherapy might boost radiation-induced immune activation, block radiation-induced immunosuppressive effects, and eliminate microscopic disease (14).

Immunotherapy is expected to be most effective when treating patients with limited disease burden (66). Additional evidence for this hypothesis comes from the results of the PACIFIC trial, in which patients with unresectable stage III NSCLC who had responded to initial chemoradiotherapy, were treated with the anti-PD-1 antibody durvalumab (67). The addition of durvalumab nearly tripled the median PFS from 5.6 months to 17.2 months and significantly improved 2-year OS from 55.6 to $66.3 \%$ ( $\mathrm{p}=$ 0.005). Furthermore, a post-hoc analysis of the KEYNOTE-001 trial demonstrated that previous radiotherapy in metastatic NSCLC patients receiving pembrolizumab significantly enhanced survival (6-months OS with radiotherapy $73 \%$ compared to $45 \%$ without) (68). Up to now, only few data are available regarding MR-guided adaptive radiotherapy for lung cancer patients $(5,25,26)$. However, several studies have demonstrated a clear benefit of CT-guided adaptive radiotherapy for optimizing target coverage and sparing healthy lung tissue and hence toxicity (69-71). MR-guided adaptive radioimmunotherapy might therefore enable further dose escalation for improving local control, while simultaneously fostering the systemic immune response against distant micrometastases.

Current studies on MR-guided adaptive pulmonary radiotherapy focus on SBRT of small central and peripheral lung lesions $(5,25,26)$. MR-guided adaptive SBRT of centrally or even ultracentrally located tumor lesions holds the promise to safely increase doses for such lesions adjacent to radiosensitive and vulnerable OARs (e.g. central airways, esophagus, heart). While local control following SBRT is usually satisfying, distant progression remains the major challenge $(72,73)$. Hence, several trials are ongoing to assess the efficacy of additional immunotherapy with SBRT for eradicating microscopic disease and fostering RT-induced immune activation in the treatment of early-stage lung cancer patients (e.g. KEYNOTE-867, PACIFIC4). MR-guided adaptive radio-immunotherapy would further allow for safe treatment of critically located pulmonary lesions with sufficiently high dose and simultaneously reduce the occurrence of new distant tumor lesions.

As discussed above, systemic responses to immunotherapy are more frequent if overall disease burden is limited. In line with 
this concept, Golden et al. analyzed the occurrence of abscopal responses in metastatic patients on chemotherapy treated with concurrent radiotherapy (35 Gy in 10 fractions) to one metastatic site and granulocyte-macrophage colony-stimulating factor (12). Interestingly, the authors described that abscopal tumor responses were more frequent in patients with limited disease sites ( $73 \%$ in patients with only three metastases). Further support for this assertion comes from another trial, in which patients with metastatic castration-resistant prostate cancer were treated with a single dose of 8 Gy to a single bone metastasis with or without ipilimumab (74). Patients with only one osseous metastasis were more likely to benefit from immunotherapy compared to those with more bone lesions. In these scenarios, MR-guided adaptive radiotherapy could enable highly precise and focused dose delivery even to critically located tumor lesions, for which conventional techniques cannot achieve sufficiently high doses, while simultaneously potentiating local effects of immunotherapy (14).

Further tumor entities like head-and-neck tumors, rectal, cervical, or bladder cancer are expected to profit from MRguided adaptive radiotherapy for not only enabling dose escalation, sparing of adjacent radiosensitive OARs but also for increasing the chance for organ preservation $(1,75-78)$. Up to now, immunotherapy is only clinically established in the treatment of metastatic tumor stages of these malignancies (79-83). Future studies are awaited to demonstrate the benefit of simultaneous radio-immunotherapy to augment local and systemic immunity and potentially reduce the risk for metastatic recurrences.

\section{MR-GUIDED RADIO-IMMUNOTHERAPY: CHALLENGES AND OPPORTUNITIES}

Preclinical models suggest a window of opportunity to combine radiotherapy and immunotherapy, and early clinical studies report favorable responses to this combination. Nevertheless, many parameters remain ill-defined and need to be resolved to fully exploit the potential of radio-immunotherapy (84). These include scheduling of both modalities, fractionation regimens, treatment volume, and response prediction. The MR-linac combines unique functionalities that can address some of these outstanding questions. With regard to the optimal sequence of both modalities, preclinical data are not conclusive and suggest a combined effect that is both tumor model and immunomodulatory agent dependent. Although results from clinical studies are still scarce, the data indicate highest (local and abscopal) efficacy when radiation shortly precedes or is given during immunotherapy $(67,68,85)$. Whether or not early radiation-induced influx of immune cells in the tumor microenvironment can be detected by MR imaging, e.g. as increased ADC values on DW MRI (86), and guide the optimal timing of immunotherapy, remains to be investigated.

Preclinical models imply that the dose per fraction is critical for the immunogenic effect of radiation and that a moderately hypofractionated regimen (range: 8-12 Gy per fraction) induces sufficient cytosolic double-stranded (ds)DNA to stimulate the cGAS-STING-Interferon type I pathway. Too high radiation doses (>12-18 Gy), however, can lead to the activation of feedback mechanisms, like the induction of the exonuclease Trexl that degrades cytosolic DNA and attenuates the cGASSTING pathway (47). This delicate biological balance between release of dsDNA and Trexl dictates dsDNA accumulation in the cytoplasm of irradiated cells, and the subsequent initiation of anti-tumor immune responses. The dose range at which such optimal conditions arise, may turn out to be tumor specific, although in general a relatively high dose per fraction (around 8 Gy) seems required. MR-guidance is an obvious tool to safely and accurately deliver these high doses of radiation and allow the identification of the most effective fractionation regimen for synergy between radiotherapy and immunotherapy.

With respect to the ideal target volume to be irradiated, MRbased functional imaging could reveal radiosensitive or radioresistant subvolumes of tumors that may benefit from differential dosing. Intriguingly, partial tumor irradiation has been shown to elicit an effective (both local and abscopal) immune response without the need to treat the entire tumor (87, 88). High precision delivery of radiation in the context of radioimmunotherapy also involves sparing of lymphoid tissue. In fact, avoiding irradiation of tumor-associated draining lymph nodes may be crucial for the integrity of the immune response. In the context of a preclinical model comparing stereotactic radiotherapy with or without elective nodal irradiation in combination with immune checkpoint blockade, it was found that an altered T-cell chemoattractant chemokine signaling resulted in reduced immune infiltration as well as in an unfavorable balance between tumoricidal and immunosuppressive immune cells (89).

A final challenge pertains to the need for robust biomarkers of response. The superior soft tissue contrast of MR increases the ability to define the location of the tumor and adjacent normal tissues and to adapt treatment based on biological and functional dynamics of both tumors and normal structures that may occur during treatment. As responses to radio-immunotherapy will vary among tumor sites, pathological subtypes and individual patients, there is a strong clinical need for solid predictors of response to treatment. In addition to tissue-based biomarkers [such as T-cell-inflamed gene-expression profile, programmed death ligand 1 (PD-L1) expression, and tumor mutational burden], imaging-based biomarkers are emerging as promising, non-invasive, and repeatable tools that may help identify patients who have a higher likelihood of response to radioimmunotherapy across a broad spectrum of tumors. The MRlinac not only allows the use of functional MR sequences, quantitative feature extraction using radiomic approaches has become available to develop such imaging-based biomarkers, including for radio-immunotherapy. Recently, a CT-based radiomic signature was developed and validated to assess tumorinfiltrating immune cells and response to immunotherapy in patients with advanced solid tumors (90). A comparable approach using MR-based information is an obvious opportunity and will be discussed in more detail in a separate contribution to this special issue. 


\section{CONCLUSIONS AND FUTURE PERSPECTIVES}

The clinical implementation of MR-guided adaptive radiotherapy has led to new approaches to compensate for poor target definition. Superior soft tissue contrast combined with real-time plan adaptation now allows to reduce margins, increase the dose per fraction and integrate functional information in highly individualized treatment plans. These features make MR-guided radiotherapy the perfect partner for immunotherapy. Radioimmunotherapy has emerged as a promising combination for the treatment of local and abscopal disease, but the conditions for synergy need further optimization. MR-guided radiotherapy could be instrumental to address some of these variables,

\section{REFERENCES}

1. Corradini S, Alongi F, Andratschke N, Belka C, Boldrini L, Cellini F, et al. MR-guidance in clinical reality: current treatment challenges and future perspectives. Radiat Oncol (2019) 14(1):92. doi: 10.1186/s13014-019-1308-y

2. Chin S, Eccles CL, McWilliam A, Chuter R, Walker E, Whitehurst P, et al. Magnetic resonance-guided radiation therapy: A review. J Med Imaging Radiat Oncol (2020) 64(1):163-77. doi: 10.1111/1754-9485.12968

3. Hall WA, Paulson ES, van der Heide UA, Fuller CD, Raaymakers BW, Lagendijk JJW, et al. The transformation of radiation oncology using realtime magnetic resonance guidance: A review. Eur J Cancer (2019) 122:42-52. doi: 10.1016/j.ejca.2019.07.021

4. Bruynzeel AME, Tetar SU, Oei SS, Senan S, Haasbeek CJA, Spoelstra FOB, et al. A prospective single-arm phase II study of stereotactic magneticresonance-guided adaptive radiotherapy for prostate cancer: Early toxicity results. Int J Radiat Oncol Biol Phys (2019) 105(5):1086-94. doi: 10.1016/ j.ijrobp.2019.08.007

5. Henke LE, Olsen JR, Contreras JA, Curcuru A, DeWees TA, Green OL, et al. Stereotactic MR-Guided Online Adaptive Radiation Therapy (SMART) for Ultracentral Thorax Malignancies: Results of a Phase 1 Trial. Adv Radiat Oncol (2019) 4(1):201-9. doi: 10.1016/j.adro.2018.10.003

6. Rudra S, Jiang N, Rosenberg SA, Olsen JR, Roach MC, Wan L, et al. Using adaptive magnetic resonance image-guided radiation therapy for treatment of inoperable pancreatic cancer. Cancer Med (2019) 8(5):2123-32. doi: 10.1002/ cam4.2100

7. Winkel D, Bol GH, Werensteijn-Honingh AM, Intven MPW, Eppinga WSC, Hes J, et al. Target coverage and dose criteria based evaluation of the first clinical 1.5T MR-linac SBRT treatments of lymph node oligometastases compared with conventional CBCT-linac treatment. Radiother Oncol (2020) 146:118-25. doi: 10.1016/j.radonc.2020.02.011

8. van Sörnsen de Koste JR, Palacios MA, Bruynzeel AME, Slotman BJ, Senan S, Lagerwaard FJ. MR-guided Gated Stereotactic Radiation Therapy Delivery for Lung, Adrenal, and Pancreatic Tumors: A Geometric Analysis. Int J Radiat Oncol Biol Physics (2018) 102(4):858-66. doi: 10.1016/j.ijrobp.2018.05.048

9. Kooreman ES, van Houdt PJ, Nowee ME, van Pelt VWJ, Tijssen RHN, Paulson ES, et al. Feasibility and accuracy of quantitative imaging on a $1.5 \mathrm{~T}$ MR-linear accelerator. Radiother Oncol (2019) 133:156-62. doi: 10.1016/ j.radonc.2019.01.011

10. Yang Y, Cao M, Sheng K, Gao Y, Chen A, Kamrava M, et al. Longitudinal diffusion MRI for treatment response assessment: Preliminary experience using an MRI-guided tri-cobalt 60 radiotherapy system. Med Phys (2016) 43 (3):1369-73. doi: 10.1118/1.4942381

11. Salama AK, Postow MA, Salama JK. Irradiation and immunotherapy: From concept to the clinic. Cancer (2016) 122(11):1659-71. doi: 10.1002/cncr.29889

12. Golden EB, Chhabra A, Chachoua A, Adams S, Donach M, Fenton-Kerimian $\mathrm{M}$, et al. Local radiotherapy and granulocyte-macrophage colony-stimulating factor to generate abscopal responses in patients with metastatic solid tumours: a proof-of-principle trial. Lancet Oncol (2015) 16(7):795-803. doi: 10.1016/S1470-2045(15)00054-6 including optimal doses and fractionation schedules, timing of both modalities, reduced delivery volumes (partial tumor irradiation; sparing draining lymph nodes), and response prediction. This requires a collaborative effort, standardization of protocols, models, and methodologies, and a systematic collection of imaging and biomaterial data.

\section{AUTHOR CONTRIBUTIONS}

All authors were involved in the conception and design of this review. JH-R, SK, MA, and MV drafted the manuscript. All authors contributed to the article and approved the submitted version.

13. Deutsch E, Chargari C, Galluzzi L, Kroemer G. Optimising efficacy and reducing toxicity of anticancer radioimmunotherapy. Lancet Oncol (2019) 20 (8):e452-e63. doi: 10.1016/S1470-2045(19)30171-8

14. Arina A, Gutiontov SI, Weichselbaum RR. Radiotherapy and Immunotherapy for Cancer: From "Systemic" to "Multisite". Clin Cancer Res (2020) 26 (12):2777-82. doi: 10.1158/1078-0432.CCR-19-2034

15. Acharya S, Fischer-Valuck BW, Kashani R, Parikh P, Yang D, Zhao T, et al. Online Magnetic Resonance Image Guided Adaptive Radiation Therapy: First Clinical Applications. Int J Radiat Oncol Biol Phys (2016) 94(2):394-403. doi: 10.1016/j.ijrobp.2015.10.015

16. Fischer-Valuck BW, Henke L, Green O, Kashani R, Acharya S, Bradley JD, et al. Two-and-a-half-year clinical experience with the world's first magnetic resonance image guided radiation therapy system. Adv Radiat Oncol (2017) 2 (3):485-93. doi: 10.1016/j.adro.2017.05.006

17. Raaymakers BW, Jurgenliemk-Schulz IM, Bol GH, Glitzner M, Kotte A, van Asselen B, et al. First patients treated with a $1.5 \mathrm{~T}$ MRI-Linac: clinical proof of concept of a high-precision, high-field MRI guided radiotherapy treatment. Phys Med Biol (2017) 62(23):L41-50. doi: 10.1088/1361-6560/ aa9517

18. Klüter S. Technical design and concept of a $0.35 \mathrm{~T}$ MR-Linac. Clin Trans Radiat Oncol (2019) 18:98-101. doi: 10.1016/j.ctro.2019.04.007

19. Winkel D, Bol GH, Kroon PS, van Asselen B, Hackett SS, WerensteijnHoningh AM, et al. Adaptive radiotherapy: The Elekta Unity MR-linac concept. Clin Trans Radiat Oncol (2019) 18:54-9. doi: 10.1016/j.ctro. 2019.04.001

20. Paulson ES, Ahunbay E, Chen X, Mickevicius NJ, Chen G-P, Schultz C, et al. 4D-MRI driven MR-guided online adaptive radiotherapy for abdominal stereotactic body radiation therapy on a high field MR-Linac: Implementation and initial clinical experience. Clin Trans Radiat Oncol (2020) 23:72-9. doi: 10.1016/j.ctro.2020.05.002

21. Green OL, Rankine LJ, Cai B, Curcuru A, Kashani R, Rodriguez V, et al. First clinical implementation of real-time, real anatomy tracking and radiation beam control. Med Phys (2018). doi: 10.1002/mp.13002

22. Hal WA, Straza MW, Chen X, Mickevicius N, Erickson B, Schultz C, et al. Initial clinical experience of Stereotactic Body Radiation Therapy (SBRT) for liver metastases, primary liver malignancy, and pancreatic cancer with $4 \mathrm{D}$ MRI based online adaptation and real-time MRI monitoring using a 1.5 Tesla MR-Linac. PloS One (2020) 15(8):e0236570. doi: 10.1371/journal. pone. 0236570

23. Rosenberg SA, Henke LE, Shaverdian N, Mittauer K, Wojcieszynski AP, Hullett CR, et al. A Multi-Institutional Experience of MR-Guided Liver Stereotactic Body Radiation Therapy. Adv Radiat Oncol (2019) 4(1):142-9. doi: 10.1016/j.adro.2018.08.005

24. Bohoudi O, Bruynzeel AME, Meijerink MR, Senan S, Slotman BJ, Palacios $\mathrm{MA}$, et al. Identification of patients with locally advanced pancreatic cancer benefitting from plan adaptation in MR-guided radiation therapy. Radiother Oncol (2019) 132:16-22. doi: 10.1016/j.radonc.2018.11.019

25. Finazzi T, Haasbeek CJA, Spoelstra FOB, Palacios MA, Admiraal MA, Bruynzeel AME, et al. Clinical Outcomes of Stereotactic MR-Guided 
Adaptive Radiation Therapy for High-Risk Lung Tumors. Int J Radiat Oncol Biol Phys (2020) 107(2):270-8. doi: 10.1016/j.ijrobp.2020.02.025

26. Finazzi T, Palacios MA, Haasbeek CJA, Admiraal MA, Spoelstra FOB, Bruynzeel AME, et al. Stereotactic MR-guided adaptive radiation therapy for peripheral lung tumors. Radiother Oncol J Eur Soc Ther Radiol Oncol (2020) 144:46-52. doi: 10.1016/j.radonc.2019.10.013

27. Henke LE, Kashani R, Hilliard J, DeWees TA, Curcuru A, Przybysz D, et al. In Silico Trial of MR-Guided Midtreatment Adaptive Planning for Hypofractionated Stereotactic Radiation Therapy in Centrally Located Thoracic Tumors. Int J Radiat Oncol Biol Phys (2018) 102(4):987-95. doi: 10.1016/j.ijrobp.2018.06.022

28. Thomas DH, Santhanam A, Kishan AU, Cao M, Lamb J, Min Y, et al. Initial clinical observations of intra- and interfractional motion variation in MRguided lung SBRT. Br J Radiol (2018) 91(1083):20170522. doi: 10.1259/ bjr.20170522

29. Bauml JM, Mick R, Ciunci C, Aggarwal C, Davis C, Evans T, et al. Pembrolizumab After Completion of Locally Ablative Therapy for Oligometastatic Non-Small Cell Lung Cancer: A Phase 2 Trial. JAMA Oncol (2019) 5(9):1283-90. doi: 10.1001/jamaoncol.2019.1449

30. Nicosia L, Sicignano G, Rigo M, Figlia V, Cuccia F, De Simone A, et al. Daily dosimetric variation between image-guided volumetric modulated arc radiotherapy and MR-guided daily adaptive radiotherapy for prostate cancer stereotactic body radiotherapy. Acta Oncol (2020) 1-7. doi: 10.1080/ 0284186x.2020.1821090

31. Tetar SU, Bruynzeel AME, Oei SS, Senan S, Fraikin T, Slotman BJ, et al. Magnetic Resonance-guided Stereotactic Radiotherapy for Localized Prostate Cancer: Final Results on Patient-reported Outcomes of a Prospective Phase 2 Study. Eur Urol Oncol (2020). doi: 10.1016/j.euo.2020.05.007

32. Nachbar M, Mönnich D, Boeke S, Gani C, Weidner N, Heinrich V, et al. Partial breast irradiation with the $1.5 \mathrm{~T}$ MR-Linac: First patient treatment and analysis of electron return and stream effects. Radiother Oncol J Eur Soc Ther Radiol Oncol (2020) 145:30-5. doi: 10.1016/j.radonc.2019.11.025

33. Acharya S, Fischer-Valuck BW, Mazur TR, Curcuru A, Sona K, Kashani R, et al. Magnetic Resonance Image Guided Radiation Therapy for External Beam Accelerated Partial-Breast Irradiation: Evaluation of Delivered Dose and Intrafractional Cavity Motion. Int J Radiat Oncol Biol Phys (2016) 96 (4):785-92. doi: 10.1016/j.ijrobp.2016.08.006

34. Henke LE, Contreras JA, Green OL, Cai B, Kim H, Roach MC, et al. Magnetic Resonance Image-Guided Radiotherapy (MRIgRT): A 4.5-Year Clinical Experience. Clin Oncol (2018) 30(11):720-7. doi: 10.1016/j.clon.2018.08.010

35. Palacios MA, Bohoudi O, Bruynzeel AME, van Sorsen de Koste JR, Cobussen P, Slotman BJ, et al. Role of Daily Plan Adaptation in MR-Guided Stereotactic Ablative Radiation Therapy for Adrenal Metastases. Int J Radiat Oncol Biol Phys (2018) 102(2):426-33. doi: 10.1016/j.ijrobp.2018.06.002

36. Henke L, Kashani R, Robinson C, Curcuru A, DeWees T, Bradley J, et al. Phase I trial of stereotactic MR-guided online adaptive radiation therapy (SMART) for the treatment of oligometastatic or unresectable primary malignancies of the abdomen. Radiother Oncol J Eur Soc Ther Radiol Oncol (2018) 126(3):519-26. doi: 10.1016/j.radonc.2017.11.032

37. Bohoudi O, Bruynzeel AME, Senan S, Cuijpers JP, Slotman BJ, Lagerwaard FJ, et al. Fast and robust online adaptive planning in stereotactic MR-guided adaptive radiation therapy (SMART) for pancreatic cancer. Radiother Oncol J Eur Soc Ther Radiol Oncol (2017) 125(3):439-44. doi: 10.1016/j.radonc. 2017.07.028

38. Gomez DR, Tang C, Zhang J, Blumenschein GRJr, Hernandez M, Lee JJ, et al. Local Consolidative Therapy Vs. Maintenance Therapy or Observation for Patients With Oligometastatic Non-Small-Cell Lung Cancer: Long-Term Results of a Multi-Institutional, Phase II, Randomized Study. J Clin Oncol (2019) 37(18):1558-65. doi: 10.1200/jco.19.00201

39. Finazzi T, van Sörnsen de Koste JR, Palacios MA, Spoelstra FOB, Slotman BJ, Haasbeek CJA, et al. Delivery of magnetic resonance-guided single-fraction stereotactic lung radiotherapy. Phys Imaging Radiat Oncol (2020) 14:17-23. doi: 10.1016/j.phro.2020.05.002

40. Kron T, Thorwarth D. Single-fraction magnetic resonance guided stereotactic radiotherapy - A game changer? Phys Imaging Radiat Oncol (2020) 14:95-6. doi: $10.1016 /$ j.phro.2020.06.003

41. Demaria S, Kawashima N, Yang AM, Devitt ML, Babb JS, Allison JP, et al. Immune-mediated inhibition of metastases after treatment with local radiation and CTLA- 4 blockade in a mouse model of breast cancer. Clin Cancer Res (2005) 11(2 Pt 1):728-34.

42. Dewan MZ, Galloway AE, Kawashima N, Dewyngaert JK, Babb JS, Formenti SC, et al. Fractionated but not single-dose radiotherapy induces an immunemediated abscopal effect when combined with anti-CTLA-4 antibody. Clin Cancer Res (2009) 15(17):5379-88. doi: 10.1158/1078-0432.Ccr-09-0265

43. Dovedi SJ, Adlard AL, Lipowska-Bhalla G, McKenna C, Jones S, Cheadle EJ, et al. Acquired resistance to fractionated radiotherapy can be overcome by concurrent PD-L1 blockade. Cancer Res (2014) 74(19):5458-68. doi: 10.1158/ 0008-5472.Can-14-1258

44. Deng L, Liang H, Xu M, Yang X, Burnette B, Arina A, et al. STING-Dependent Cytosolic DNA Sensing Promotes Radiation-Induced Type I InterferonDependent Antitumor Immunity in Immunogenic Tumors. Immunity (2014) 41(5):843-52. doi: 10.1016/j.immuni.2014.10.019

45. Park SS, Dong H, Liu X, Harrington SM, Krco CJ, Grams MP, et al. PD-1 Restrains Radiotherapy-Induced Abscopal Effect. Cancer Immunol Res (2015) 3(6):610-9. doi: 10.1158/2326-6066.CIR-14-0138

46. Dovedi SJ, Cheadle EJ, Popple AL, Poon E, Morrow M, Stewart R, et al. Fractionated Radiation Therapy Stimulates Antitumor Immunity Mediated by Both Resident and Infiltrating Polyclonal T-cell Populations when Combined with PD-1 Blockade. Clin Cancer Res (2017) 23(18):5514-26. doi: 10.1158/ 1078-0432.Ccr-16-1673

47. Vanpouille-Box C, Alard A, Aryankalayil MJ, Sarfraz Y, Diamond JM, Schneider RJ, et al. DNA exonuclease Trex1 regulates radiotherapy-induced tumour immunogenicity. Nat Commun (2017) 8:15618. doi: 10.1038/ ncomms 15618

48. Chen Q, Sun L, Chen ZJ. Regulation and function of the cGAS-STING pathway of cytosolic DNA sensing. Nat Immunol (2016) 17(10):1142-9. doi: $10.1038 /$ ni.3558

49. Liang H, Deng L, Hou Y, Meng X, Huang X, Rao E, et al. Host STINGdependent MDSC mobilization drives extrinsic radiation resistance. Nat Commun (2017) 8(1):1736. doi: 10.1038/s41467-017-01566-5

50. Verhaegen F, Granton P, Tryggestad E. Small animal radiotherapy research platforms. Phys Med Biol (2011) 56(12):R55-83. doi: 10.1088/0031-9155/56/ 12/R01

51. Herter-Sprie GS, Korideck H, Christensen CL, Herter JM, Rhee K, Berbeco RI, et al. Image-guided radiotherapy platform using single nodule conditional lung cancer mouse models. Nat Commun (2014) 5:5870. doi: 10.1038/ ncomms 6870

52. Dobiasch S, Kampfer S, Habermehl D, Duma MN, Felix K, Strauss A, et al. MRI-based high-precision irradiation in an orthotopic pancreatic tumor mouse model : A treatment planning study. Strahlenther Onkol (2018) 194 (10):944-52. doi: 10.1007/s00066-018-1326-y

53. Kersemans V, Beech JS, Gilchrist S, Kinchesh P, Allen PD, Thompson J, et al. An efficient and robust MRI-guided radiotherapy planning approach for targeting abdominal organs and tumours in the mouse. PloS One (2017) 12(4): e0176693. doi: 10.1371/journal.pone. 0176693

54. Corroyer-Dulmont A, Falzone N, Kersemans V, Thompson J, Hill M, Allen $\mathrm{PD}$, et al. MRI-guided radiotherapy of the SK-N-SH neuroblastoma xenograft model using a small animal radiation research platform. Br J Radiol (2017) 90 (1069):20160427. doi: 10.1259/bjr.20160427

55. Sosa Iglesias V, van Hoof SJ, Vaniqui A, Schyns LE, Lieuwes N, Yaromina A, et al. An orthotopic non-small cell lung cancer model for image-guided small animal radiotherapy platforms. Br J Radiol (2019) 92(1095):20180476. doi: 10.1259/bjr.20180476

56. Butterworth KT, Ghita M, McMahon SJ, McGarry CK, Griffin RJ, Hounsell $\mathrm{AR}$, et al. Modelling responses to spatially fractionated radiation fields using preclinical image-guided radiotherapy. Br J Radiol (2017) 90(1069):20160485. doi: $10.1259 /$ bjr. 20160485

57. Mole RH. Whole body irradiation; radiobiology or medicine?10.1259/00071285-26-305-234. Br J Radiol (1953) 26(305):234-41. doi: 10.1259/0007-128526-305-234

58. Reynders K, Illidge T, Siva S, Chang JY, De Ruysscher D. The abscopal effect of local radiotherapy: using immunotherapy to make a rare event clinically relevant. Cancer Treat Rev (2015) 41(6):503-10. doi: 10.1016/j.ctrv. 2015.03.011

59. Ost P, Reynders D, Decaestecker K, Fonteyne V, Lumen N, Bruycker AD, et al. Surveillance or Metastasis-Directed Therapy for Oligometastatic Prostate 
Cancer Recurrence: A Prospective, Randomized, Multicenter Phase II Trial. J Clin Oncol (2018) 36(5):446-53. doi: 10.1200/jco.2017.75.4853

60. Palma DA, Olson R, Harrow S, Gaede S, Louie AV, Haasbeek C, et al. Stereotactic ablative radiotherapy versus standard of care palliative treatment in patients with oligometastatic cancers (SABR-COMET): a randomised, phase 2, open-label trial. Lancet (2019) 393(10185):2051-8. doi: 10.1016/ S0140-6736(18)32487-5

61. Iyengar P, Wardak Z, Gerber DE, Tumati V, Ahn C, Hughes RS, et al. Consolidative Radiotherapy for Limited Metastatic Non-Small-Cell Lung Cancer: A Phase 2 Randomized Clinical Trial. JAMA Oncol (2018) 4(1): e173501-e. doi: 10.1001/jamaoncol.2017.3501

62. Palma DA, Olson R, Harrow S, Gaede S, Louie AV, Haasbeek C, et al. Stereotactic Ablative Radiotherapy for the Comprehensive Treatment of Oligometastatic Cancers: Long-Term Results of the SABR-COMET Phase II Randomized Trial. J Clin Oncol (2020) 38(25):2830-8. doi: 10.1200/ jco. 20.00818

63. Theelen WSME, Peulen HMU, Lalezari F, van der Noort V, de Vries JF, Aerts JGJV, et al. Effect of Pembrolizumab After Stereotactic Body Radiotherapy vs Pembrolizumab Alone on Tumor Response in Patients With Advanced NonSmall Cell Lung Cancer: Results of the PEMBRO-RT Phase 2 Randomized Clinical Trial. JAMA Oncol (2019) 5(9):1276-82. doi: 10.1001/ jamaoncol.2019.1478

64. Feldman AM, Modh A, Glide-Hurst C, Chetty IJ, Movsas B. Real-time Magnetic Resonance-guided Liver Stereotactic Body Radiation Therapy: An Institutional Report Using a Magnetic Resonance-Linac System. Cureus (2019) 11(9):e5774. doi: 10.7759/cureus.5774

65. Llorente R, Spieler BO, Victoria J, Takita C, Yechieli R, Ford JC, et al. MRIguided stereotactic ablative radiation therapy of spinal bone metastases: a preliminary experience. Br J Radiol (2020) 93(1105):20190655. doi: 10.1259/ bjr. 20190655

66. Pitroda SP, Chmura SJ, Weichselbaum RR. Integration of radiotherapy and immunotherapy for treatment of oligometastases. Lancet Oncol (2019) 20(8): e434-e42. doi: 10.1016/S1470-2045(19)30157-3

67. Antonia SJ, Villegas A, Daniel D, Vicente D, Murakami S, Hui R, et al. Durvalumab after Chemoradiotherapy in Stage III Non-Small-Cell Lung Cancer. N Engl J Med (2017) 377(20):1919-29. doi: 10.1056/NEJMoa1709937

68. Shaverdian N, Lisberg AE, Bornazyan K, Veruttipong D, Goldman JW, Formenti SC, et al. Previous radiotherapy and the clinical activity and toxicity of pembrolizumab in the treatment of non-small-cell lung cancer: a secondary analysis of the KEYNOTE-001 phase 1 trial. Lancet Oncol (2017) 18 (7):895-903. doi: 10.1016/S1470-2045(17)30380-7

69. Guckenberger M, Richter A, Wilbert J, Flentje M, Partridge M. Adaptive radiotherapy for locally advanced non-small-cell lung cancer does not underdose the microscopic disease and has the potential to increase tumor control. Int J Radiat Oncol Biol Phys (2011) 81(4):e275-82. doi: 10.1016/ j.ijrobp.2011.01.067

70. Guckenberger M, Wilbert J, Richter A, Baier K, Flentje M. Potential of adaptive radiotherapy to escalate the radiation dose in combined radiochemotherapy for locally advanced non-small cell lung cancer. Int $J$ Radiat Oncol Biol Phys (2011) 79(3):901-8. doi: 10.1016/j.ijrobp.2010.04.050

71. Møller DS, Holt MI, Alber M, Tvilum M, Khalil AA, Knap MM, et al. Adaptive radiotherapy for advanced lung cancer ensures target coverage and decreases lung dose. Radiother Oncol (2016) 121(1):32-8. doi: 10.1016/ j.radonc.2016.08.019

72. Cushman TR, Gomez D, Kumar R, Likacheva A, Chang JY, Cadena AP, et al. Combining radiation plus immunotherapy to improve systemic immune response. J Thorac Dis (2018) 10(Suppl 3):S468-S79. doi: 10.21037/ jtd.2018.01.130

73. Lin AJ, Roach M, Bradley J, Robinson C. Combining stereotactic body radiation therapy with immunotherapy: current data and future directions. Transl Lung Cancer Res (2019) 8(1):107-15. doi: 10.21037/ tlcr.2018.08.16

74. Kwon ED, Drake CG, Scher HI, Fizazi K, Bossi A, van den Eertwegh AJ, et al. Ipilimumab versus placebo after radiotherapy in patients with metastatic castration-resistant prostate cancer that had progressed after docetaxel chemotherapy (CA184-043): a multicentre, randomised, double-blind, phase 3 trial. Lancet Oncol (2014) 15(7):700-12. doi: 10.1016/S1470-2045 (14)70189-5
75. Bahig H, Yuan Y, Mohamed ASR, Brock KK, Ng SP, Wang J, et al. Magnetic Resonance-based Response Assessment and Dose Adaptation in Human Papilloma Virus Positive Tumors of the Oropharynx treated with Radiotherapy (MR-ADAPTOR): An R-IDEAL stage 2a-2b/Bayesian phase II trial. Clin Transl Radiat Oncol (2018) 13:19-23. doi: 10.1016/j.ctro. 2018.08.003

76. Mohamed ASR, Bahig H, Aristophanous M, Blanchard P, Kamal M, Ding Y, et al. Prospective in silico study of the feasibility and dosimetric advantages of MRI-guided dose adaptation for human papillomavirus positive oropharyngeal cancer patients compared with standard IMRT. Clin Transl Radiat Oncol (2018) 11:11-8. doi: 10.1016/j.ctro.2018.04.005

77. Chiloiro G, Boldrini L, Meldolesi E, Re A, Cellini F, Cusumano D, et al. MRguided radiotherapy in rectal cancer: First clinical experience of an innovative technology. Clin Trans Radiat Oncol (2019) 18:80-6. doi: 10.1016/ j.ctro.2019.04.006

78. Gani C, Boldrini L, Valentini V. Online MR guided radiotherapy for rectal cancer. New opportunities. Clin Trans Radiat Oncol (2019) 18:66-7. doi: 10.1016/j.ctro.2019.04.005

79. Ganesh K, Stadler ZK, Cercek A, Mendelsohn RB, Shia J, Segal NH, et al. Immunotherapy in colorectal cancer: rationale, challenges and potential. Nat Rev Gastroenterol Hepatol (2019) 16(6):361-75. doi: 10.1038/s41575-0190126-x

80. Wołącewicz M, Hrynkiewicz R, Grywalska E, Suchojad T, Leksowski T, Roliński J, et al. Immunotherapy in Bladder Cancer: Current Methods and Future Perspectives. Cancers (Basel) (2020) 12(5):1181. doi: 10.3390/ cancers 12051181

81. Cohen EEW, Soulières D, Le Tourneau C, Dinis J, Licitra L, Ahn M-J, et al. Pembrolizumab versus methotrexate, docetaxel, or cetuximab for recurrent or metastatic head-and-neck squamous cell carcinoma (KEYNOTE-040): a randomised, open-label, phase 3 study. Lancet (2019) 393(10167):156-67. doi: 10.1016/S0140-6736(18)31999-8

82. Bauml JM, Aggarwal C, Cohen RB. Immunotherapy for head and neck cancer: where are we now and where are we going? Ann Transl Med (2019) 7(Suppl 3):S75-S. doi: 10.21037/atm.2019.03.58

83. Cramer JD, Burtness B, Ferris RL. Immunotherapy for head and neck cancer: Recent advances and future directions. Oral Oncol (2019) 99:104460. doi: 10.1016/j.oraloncology.2019.104460

84. Wang Y, Deng W, Li N, Neri S, Sharma A, Jiang W, et al. Combining Immunotherapy and Radiotherapy for Cancer Treatment: Current Challenges and Future Directions. Front Pharmacol (2018) 9:185. doi: 10.3389/ fphar.2018.00185

85. Formenti SC, Rudqvist NP, Golden E, Cooper B, Wennerberg E, Lhuillier C, et al. Radiotherapy induces responses of lung cancer to CTLA-4 blockade. Nat Med (2018) 24(12):1845-51. doi: 10.1038/s41591018-0232-2

86. Chung YH, Yu CF, Chiu SC, Chiu H, Hsu ST, Wu CR, et al. Diffusionweighted MRI and (18)F-FDG PET correlation with immunity in early radiotherapy response in BNL hepatocellular carcinoma mouse model: timeline validation. Eur J Nucl Med Mol Imaging (2019) 46(8):1733-44. doi: 10.1007/s00259-019-04318-3

87. Markovsky E, Budhu S, Samstein RM, Li H, Russell J, Zhang Z, et al. An Antitumor Immune Response Is Evoked by Partial-Volume Single-Dose Radiation in 2 Murine Models. Int J Radiat Oncol Biol Phys (2019) 103 (3):697-708. doi: 10.1016/j.ijrobp.2018.10.009

88. Tubin S, Popper HH, Brcic L. Novel stereotactic body radiation therapy (SBRT)-based partial tumor irradiation targeting hypoxic segment of bulky tumors (SBRT-PATHY): improvement of the radiotherapy outcome by exploiting the bystander and abscopal effects. Radiat Oncol (2019) 14(1):21. doi: 10.1186/s13014-019-1227-y

89. Marciscano AE, Ghasemzadeh A, Nirschl TR, Theodros D, Kochel CM, Francica BJ, et al. Elective Nodal Irradiation Attenuates the Combinatorial Efficacy of Stereotactic Radiation Therapy and Immunotherapy. Clin Cancer Res (2018) 24(20):5058-71. doi: 10.1158/1078-0432.CCR-17-3427

90. Sun R, Limkin EJ, Vakalopoulou M, Dercle L, Champiat S, Han SR, et al. A radiomics approach to assess tumour-infiltrating $\mathrm{CD} 8$ cells and response to anti-PD-1 or anti-PD-L1 immunotherapy: an imaging biomarker, retrospective multicohort study. Lancet Oncol (2018) 19(9):1180-91. doi: $10.1016 / \mathrm{s} 1470-2045(18) 30413-3$ 
Conflict of Interest: JH-R and SK received speaker fees and travel reimbursement from ViewRay Inc. JH-R received travel reimbursement from IntraOP Medical and Elekta Instrument AB outside the submitted work. JH-R received a grant from IntraOP Medical outside the submitted work. JD received grants from CRI-The Clinical Research Institute GmbH, View Ray Inc., Accuray International, Accuray Incorporated, RaySearch Laboratories AB, Vision RT limited, Astellas Pharma $\mathrm{GmbH}$, Merck Serono GmbH, Astra Zeneca GmbH, Solution Akademie GmbH, Ergomed PLC Surrey Research Park, Siemens Healthcare GmbH, Quintiles $\mathrm{GmbH}$, Pharmaceutical Research Associates $\mathrm{GmbH}$, Boehringer Ingelheim Pharma GmbH Co, PTW-Freiburg Dr. Pychlau GmbH, Nanobiotix A.A., as well as IntraOP Medical outside the submitted work. JD has a research agreement with ViewRay Inc. and is member of the Scientific Advisory Board of ViewRay Inc. (Oakwood, USA). MV received grant support from Hoffmann-LaRoche, AstraZeneca, AbbVie Inc., the Dutch Colorectal Cancer Group, and the Dutch
Cancer Society outside the submitted work. MV has a research agreement with Elekta Ltd.

The remaining authors declare that the research was conducted in the absence of any commercial or financial relationships that could be construed as a potential conflict of interest.

Copyright (C) 2021 Hörner-Rieber, Klüter, Debus, Adema, Ansems and Verheij. This is an open-access article distributed under the terms of the Creative Commons Attribution License (CC BY). The use, distribution or reproduction in other forums is permitted, provided the original author(s) and the copyright owner(s) are credited and that the original publication in this journal is cited, in accordance with accepted academic practice. No use, distribution or reproduction is permitted which does not comply with these terms. 